\title{
Pathophysiology and Grading of the Ventral Displacement of Dorsal Spinal Cord Spectrum
}

\author{
Amarnath Chellathurai, Suhasini Balasubramaniam, \\ Sathyan Gnanasihamani, Sukumar Ramasamy, Jayamani Durairajan \\ Department of Radiodiagnosis, Stanley Medical College, Chennai, India
}

\begin{abstract}
Study Design: A retrospective study of the ventral displacement of dorsal spinal cord (VDDSC) spectrum pathophysiology and grading.

Purpose: This study aimed at examining the pathophysiology of VDDSC between D3 and D7, using magnetic resonance imaging (MRI) correlation and severity grading.

Overview of Literature: The pathologies that lead to VDDSC were previously discussed in various articles. We attempted to group these pathological conditions under a single spectrum, and grade them according to their severity.

Methods: We reviewed the MRI images of the dorsal spines of 1,350 patients over a period of 4 years (February 2013-February 2017); all MRI images were analyzed by two experienced radiologists.

Results: Of the 1,350 patients, 28 exhibited VDDSC between D3 and D7. Additional findings included ventral transdural herniation of the spinal cord $(n=10)$, anterior spinal cord adhesion $(n=7)$, arachnoid web $(n=6)$, and arachnoid cyst $(n=5)$.

Conclusions: We grouped the pathologies that lead to VDDSC at the thoracic level into a single spectrum of varying severity and graded VDDSC, from mild to severe.
\end{abstract}

Keywords: Dorsal spinal cord; Ventral displacement; Adhesion; Arachnoid web; Arachnoid cyst

\section{Introduction}

Ventral displacement of dorsal spinal cord (VDDSC) was previously considered a rare condition. Presently, it is increasingly identified because of improved diagnostic modalities such as magnetic resonance imaging (MRI). Despite increased diagnosis, its pathogenesis, natural history, treatment, and prognosis still remains controversial.

VDDSC may be congenital or acquired. Ventral transdural herniation of spinal cord, anterior spinal cord adhe- sion, arachnoid webs, and arachnoid cysts cause VDDSC. VDDSC is the most common cause of progressive myelopathy. The earliest symptoms include numbness, decreased temperature sensation in the lower limbs, and back pain. In long-standing cases, it leads to gait disturbances, bladder and bowel incontinence, and paraparesis. Hence, VDDSC should be diagnosed and treated as early as possible. The study aimed at examining the pathophysiology of VDDSC between D3 and D7 using MRI imaging correlation with severity grading.

Received Apr 14, 2017; Revised Jun 10, 2017; Accepted Jun 12, 2017

Corresponding author: Suhasini Balasubramaniam

Department of Radiodiagnosis, Govt Stanley Medical College, Old Jail Road, Old Washermanpet, Chennai 600001, India

Tel: +91-9884877622, Fax: +91-04425282350,E-mail: amarrd02@yahoo.co.in 


\section{Materials and Methods}

This retrospective study included 1,350 patients (age, 1060 years old; 584 males, 766 females) who were referred to our department of radiodiagnosis, Stanley Medical Collegez for imaging over a period of 4 years (February 2013-February 2017). This study was approved by the Institutional Review Board of Stanley Medical Collegez (IRB approval no., 0603107-10003; Reg. no., Ecr/131/inst/ TN/2013). Patients with or without specific clinical neurological symptoms were included, whereas patients with a history of traumas and surgeries were excluded from the study. Most cases were incidentally found during lumbar and cervical spine MRI examinations, which usually included whole spine T2 sagittal images as part of the scanning protocol. Because it was a retrospective study, the follow-up images or surgical details of the patients were not included.

\section{Magnetic resonance imaging protocol}

Spinal MRIs were performed using the Siemens Magnetom Aera 48 Channel 1.5 Tesla Machine (Siemens Healthcare GmbH, Erlangen, Germany) according to the standard protocol at our institution. T1W and T2W sagittal and axial images and phase contrast images were obtained for analysis.

Scan parameters used were as follows: (1) sagittal T1weighted spin-echo (repetition time/echo time, 500/7.7 msec; section thickness, $4 \mathrm{~mm}$; field of view, $230 \times 230$ $\mathrm{mm}$ ); (2) sagittal T2-weighted spin-echo (repetition time/ echo time, 2,000/87 msec; section thickness, $4 \mathrm{~mm}$; field of view, $180 \times 180 \mathrm{~mm}$ ); (3) axial T1-weighted spin-echo (repetition time/echo time, 400/8.8 msec; section thickness, $4 \mathrm{~mm}$; field of view $230 \times 230 \mathrm{~mm}$ ); and (4) axial T2- weighted spin-echo (repetition time/echo time, 4,500/99 msec; section thickness, $4 \mathrm{~mm}$; field of view, $230 \times 230$ $\mathrm{mm})$.

All MRI images were analyzed by two experienced radiologists. They noted the presence of VDDSC in 28 patients who were subsequently included in the study. The imaging criteria used to group the various pathological entities are described in detail in Table 1 [1-4].

\section{Results}

The retrospective study cohort comprised 584 males and 766 females (age, 10-60 years). We observed symmetric VDDSC with herniation due to an anterior dural defect in ten patients, VDDSC due to adhesion without herniation in seven patients, symmetric VDDSC due to a posterior arachnoid cyst in five patients, and posterior indentation of VDDSC due to arachnoid web in six patients. Patient details and imaging findings are described in Table 2.

\section{Discussion}

Given our existing knowledge of the normal physiological processes that predispose the thoracic spinal cord to anterior displacement $[2,5]$ and pathological conditions that cause VDDSC, we attempted to group VDDSC into various severity stages. MRI was the single most useful diagnostic modality of choice for VDDSC diagnosis.

The spinal cord is fixed by paired denticulate ligaments that run along the spinal canal (Fig. 1A, B). These fine reinforcements laterally extend from the spinal cord pia mater to the dura mater. The reinforcements provide stability to the cord during movement [6]. The fibrous septum posticum divides the posterior aspect of the subarachnoid space by running opposite the posterior median sulcus

Table 1. Imaging criteria for vddsc spectrum

1. Spinal cord herniation

2. Cord adhesion

3. Arachnoid web/membrane

4. Arachnoid cyst

Focal anterior spinal cord displacement with herniation outside the dura margins and an anterior C-shaped kink of the cord [1-3].

Focal anterior spinal cord displacement with an asymmetric dented appearance deformity along the posterior aspect of the cord [2].

Anterior spinal cord displacement with focal indentation in the posterior aspect of upper thoracic spinal cordscalpel sign [4].

Wide, smooth, and symmetric anterior spinal cord displacement with cerebrospinal fluid loss pulsation artifact in the posterior subarachnoid space [2]. 


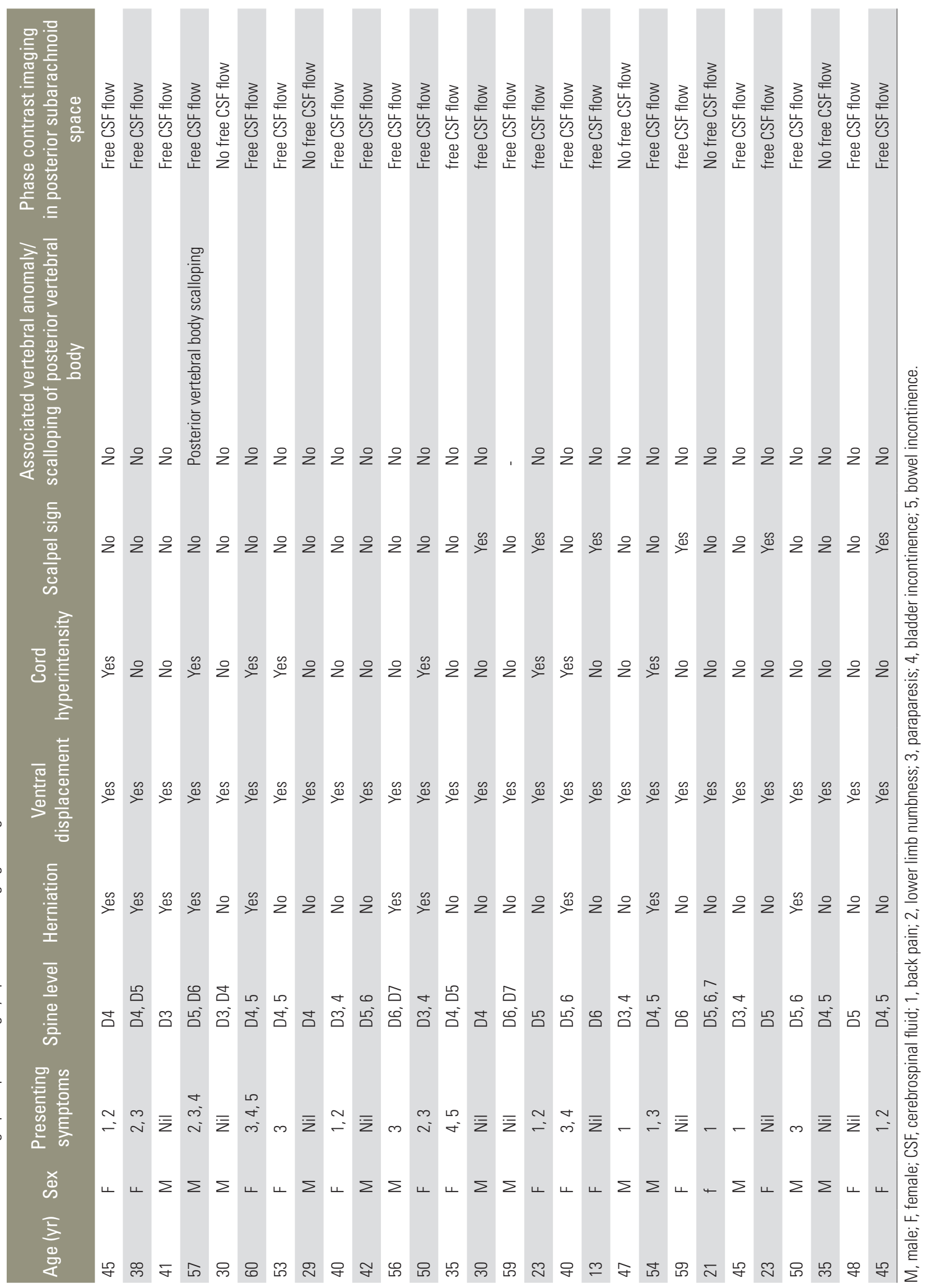


Table 3. Grading of vddsc based on severity

\begin{tabular}{llcc} 
Severity grade & Pathology & No. of patients & \% of occurrence in the study group \\
\hline 1 & Arachnoid web & 6 & 21 \\
2 & Arachnoid cyst & 5 & 18 \\
\hline 3 & Cord adhesion & 7 & 25 \\
4 & Cord herniation & 10 & 36 \\
\hline Total & & 28 & 100 \\
\hline
\end{tabular}
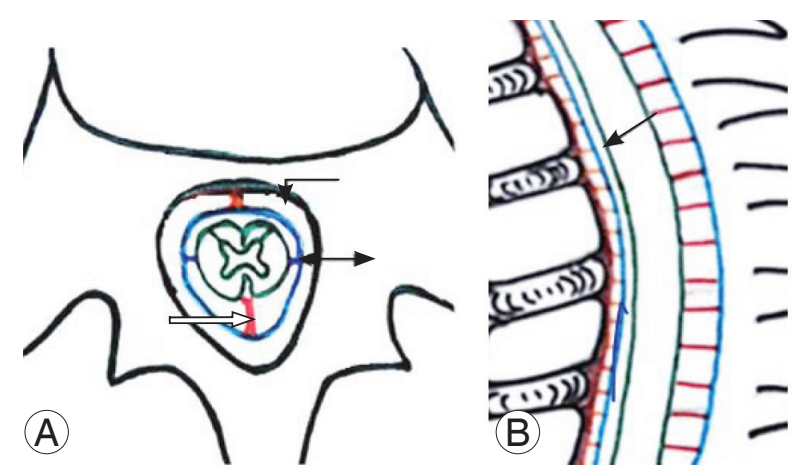

Fig. 1. (A, B) Schematic diagram of sagittal and axial sections of the dorsal spine showing normal anterior position of spinal cord (arrow) due to dorsal kyphosis. Hoffman ligament (curved arrow), denticulate ligament (double arrow), and septum posticum (open arrow).

$[6,7]$. The septum posticum is best developed in the upper thoracic spine and is believed to provide additional dorsal stability to the thoracic cord (Fig. 1A, B). Normally, the dorsal spinal cord is anteriorly positioned because of normal kyphosis and other physiological parameters, as mentioned below (Fig. 1A, B).

\section{Arachnoid web}

An arachnoid web/membrane is a milder form of VDDSC. Here, the septum posticum is fenestrated during development. Superimposed chronic degenerative disintegration of the septum may induce membrane formation, thick floating remnants, and adhesions in a subset of the population. Thick membranous remnants of the septum may result in arachnoid membrane/web formation (Fig. $2 \mathrm{~A}, \mathrm{~B})$. Arachnoid webs are likely to represent focal bands of arachnoiditis, but may be difficult to visualize on routine MRI sequences [6].

MRI findings include a mass effect on the posterior aspect of the thoracic spinal cord, causing flattening of the posterior margin [7]. There is also a mild ventral displacement of the thoracic cord at the involved levels without evidence of any mass or ventral cord herniation [8]. Cere-

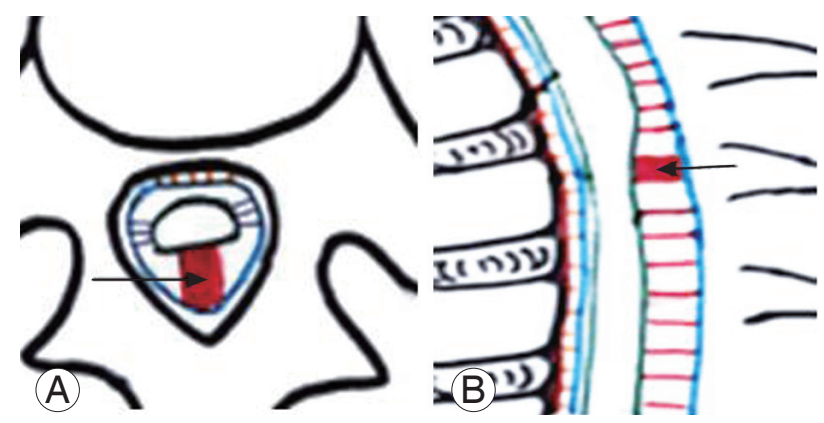

Fig. 2. (A, B) Schematic diagram of sagittal and axial sections of dorsal spine showing mild ventral displacement due to thickening of the septum posticum (straight arrow) with scalpel sign-arachnoid web. (C) Magnetic resonance imaging T2 sagittal section shows anterior cord displacement with scalpel sign at the D4 vertebral level (arrow).

brospinal fluid (CSF) flow is preserved, as is the presence of CSF flow artifacts in the posterior subarachnoid space at that level. VDDSC, due to posterior focal indentation of the cord by the arachnoid bands, produces a 'scalpel sign' (Fig. 2C) [4]. There is a widening of the posterior subarachnoid space, causing progressive alteration of CSF flow dynamics. Adhesive membranes and turbulent CSF flow lead to cord compression. Long-standing arachnoid webs may result in syringohydromyelia; however, most patients are asymptomatic and the displacement is incidentally detected.

\section{Arachnoid cysts}

Arachnoid cysts are intra-arachnoid fluid filled cystic lesions. The presence of an arachnoid cyst and VDDSC is correlated [9], the most common location being the posterior subarachnoid space of the dorsal spine $[2,9]$. Intramural spinal arachnoid cysts appear to result from arachnoid trabeculae alterations. They may also occur secondary to CSF inflow into the septum posticum or through the arachnoid diverticula [4]. Intradural arachnoid cysts can thus be attributed to septum posticum derangement. Cysts cause a highly severe VDDSC compared 

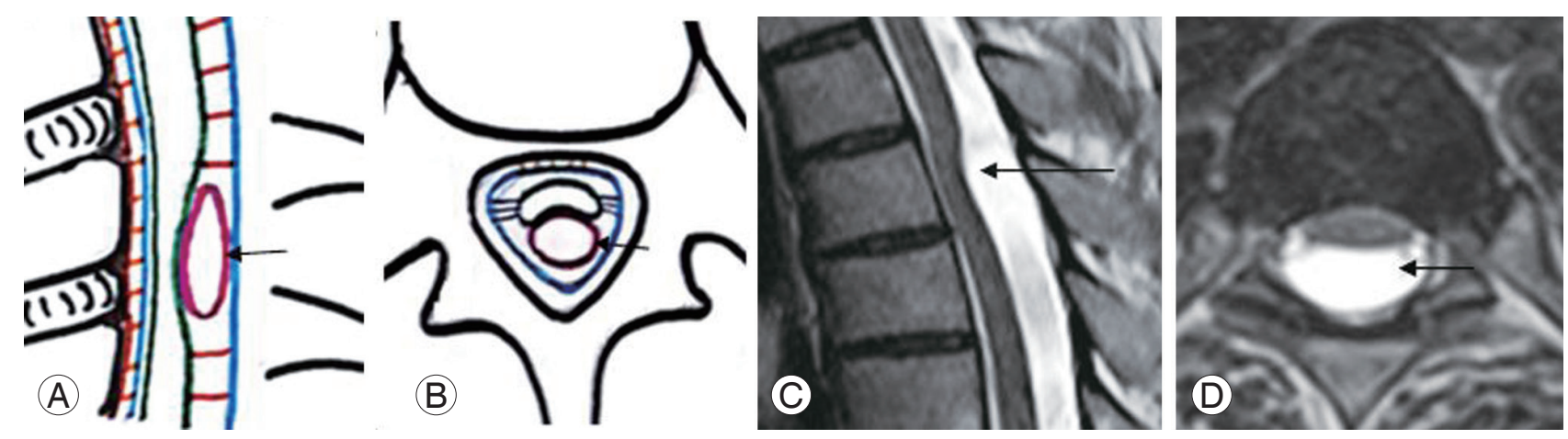

Fig. 3. (A, B) Schematic diagram of sagittal and axial sections of the dorsal spine showing moderate ventral displacement due to arachnoid cyst formation (arrow). (C, D) Magnetic resonance imaging T2 sagittal and axial sections of a 38-year-old male patient with arachnoid cyst (straight arrow) causing cord compression with smooth ventral displacement at the D3 and D4 vertebral level.
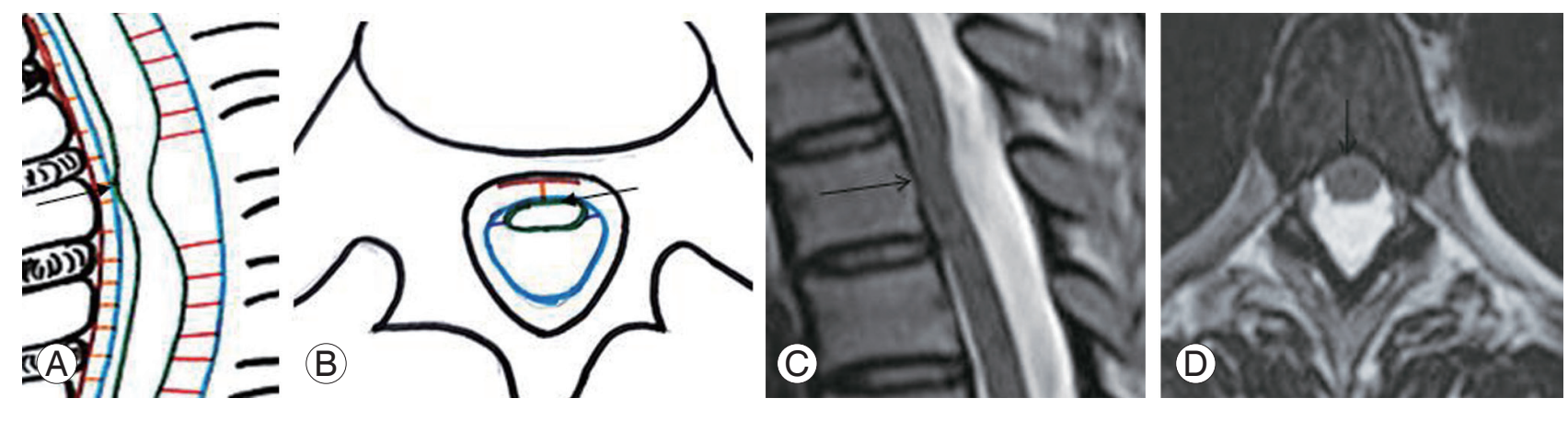

Fig. 4. (A, B) Schematic diagram and (C, D) magnetic resonance imaging spine T2 weighted sagittal and axial sections of the dorsal spine show severe ventral displacement of dorsal spinal cord due to adhesion (straight arrow) to the ventral dura with a widened posterior subarachnoid space, without cord herniation.

with arachnoid membranes/webs. Hence, cysts can be grouped under moderate severity VDDSC.

Posterior intradural arachnoid cysts are diagnosed on the basis of their unique MRI findings. They appear as uniform fluid intensities on all MRI sequences, with loss of normal CSF pulsation artifacts during phase contrast imaging, with smooth symmetric anterior spinal cord displacements (Fig. 3A-D) [2].

\section{Cord adhesion and herniation}

Ventral spinal cord adhesion is defined as focal VDDSC without spinal cord herniation or intradural mass [3]. Anterior spinal cord herniation is the herniation of the spinal cord caused by a defect in the anterior dura mater [1].

MRI findings associated with ventral cord adhesion include focal VDDSC involving one or two vertebral segments in the upper thoracic spine and focal widening of the posterior subarachnoid space (Fig. 4A-D). Normal CSF flow artifacts are noted throughout the affected spinal cord levels without cord herniation [2]. Similar findings were observed in seven patients from our study group. Of these, one demonstrated a focal T2 cord hyperintensity.

The MRI findings of ventral cord herniation are typical, with cord herniation through the anterolateral or anterior dural defects. On sagittal section, an anterior C-shaped kink of the cord can be seen with an irregular posterior margin and expansion of the dorsal subarachnoid space (Fig. 5A, C). On axial section, the herniated cord is visible as a protrusion through the ventral dura mater with no intervening CSF (Fig. 5B, D). The herniated cord may be thinned out and may show signal changes due to cord atrophy. The presence of normal CSF pulsation artifacts and free CSF flow in phase contrast pulse cine MRI differentiates it from an arachnoid cyst. In the present study, 10 patients demonstrated spinal cord herniation through dural defects between the D3 and D7 vertebral levels. A focal widening of the dorsal subarachnoid space in all patients and focal cord hyperintensity in six patients was also observed. Ventral cord herniation caused posterior vertebral 

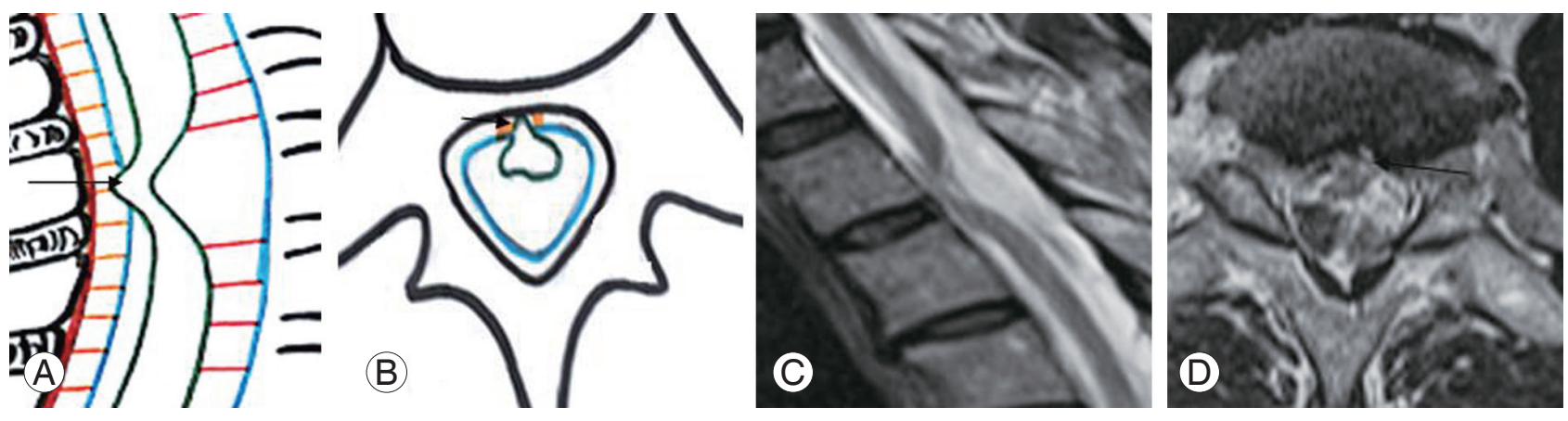

Fig. 5. (A, B) Schematic diagram shows (C, D) magnetic resonance imaging spine T2 weighted sagittal and axial sections of dorsal spine with severe asymmetric ventral displacement of the cord with herniation (straight arrow).

body scalloping in one patient.

Ventral spinal cord herniation and cord adhesion are the two ends of the same pathology, with cord adhesion being a less-severe form [8]. Because cord adhesion and herniation cause significant anterior displacement of the dorsal cord with pathological changes in the ventral dura, these entities can be graded as severe forms of VDDSC.

Despite several studies being conducted, the pathogenesis of these conditions still remains unclear. One study reports that the traction force generated by the anteriorlyplaced Hoffman ligaments along with normal physiological kyphosis with normal ventral motion secondary to cardiac pulsations and pulmonary actions at that level, and the biomechanical impact of flexion extension movements on the thoracic spinal cord tend to pull the cord, causing VDDSC and subsequently leading to cord adhesion and herniation (more commonly in the thoracic level) $[2,5,10]$. This keeps the cord in contact with the ventral dura. If this persists over time, it leads to irritation and inflammation [11], which ultimately ends in cord adherence over the ventral dura. If the process persists, it may eventually cause a ventral dural tear, resulting in a dural defect. CSF freely flows through the dural defects, leading to defect enlargement and tissue reaction. Dural defect widening and tissue reaction lead to spinal cord protrusion at the vertebral body [3].

Isu et al. [12] postulated that dorsal arachnoid cysts exert pressure effects over the spinal cord, and along with physiological events, lead to spinal cord adhesion and subsequent herniation, as described above [13]. Another study proposes an inflammatory etiology of spinal cord herniation at the intervertebral disk level. Intervertebral disk inflammation leads to adhesion with the adjacent ventral dura because of inflammatory thickening of the dentate ligament and arachnoid membrane. Subsequent resorption of the disk results in pathological cord displacement and eventual cord herniation [9].

Parmar et al. [3] stated that when a lax denticulate ligament fails to stabilize spinal cord movement during cardiac and respiratory actions, the spinal cord may anteriorly displace. Congenital dural deficiencies and ventral dural duplications can also lead to cord adhesion and herniation [14].

According to previous studies and the present one, dural defects along with physiological events alone cannot cause spinal cord adhesion and herniation; rather, spinal cord adhesions and herniations at the vertebral body and intervertebral disk levels can be explained by the following mechanisms.

\section{Potential theories explaining dorsal spinal cord adhe- sion}

1) Anterior traction theory

Anterior Hoffman ligament traction, along with physiological events, may not anteriorly displace the cord. Arachnoid inflammation and adhesion are essential for spinal cord adhesion (Fig. 6A).

2) Lax lateral denticulate ligament theory

Lax denticulate ligament leads to anterior displacement of the spinal cord, thereby leading to repetitive friction between the arachnoid membrane and dura mater, resulting in inflammation, adhesion, and dural tears (Fig. 6B).

\section{3) Posterior compression theory}

The posterior compression theory states that thickened septum posticum cause an indentation on the posterior aspect of the spinal cord. Unfortunately, no followup spine images detailing progression of posterior cord compression to adhesion are available. We proposed that 


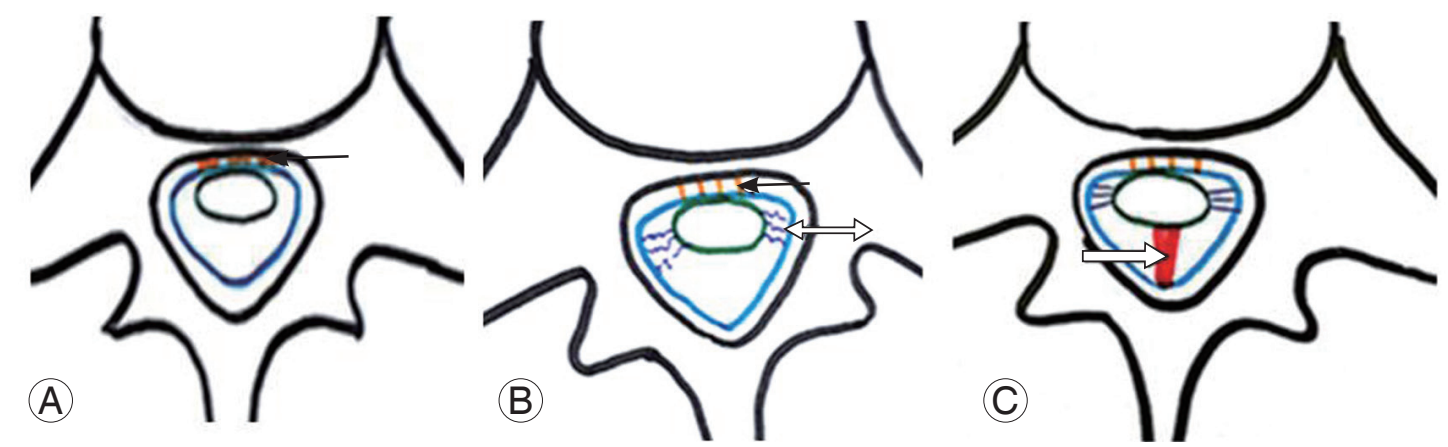

Fig. 6. (A-C) Schematic diagram showing ventral displacement of dorsal spinal cord in cord adhesions: mechanisms showing ventral displacement of the spinal cord due to arachnoid adhesions (straight arrow), lax denticulate ligament (double arrow), and septum posticum (open arrow), respectively.
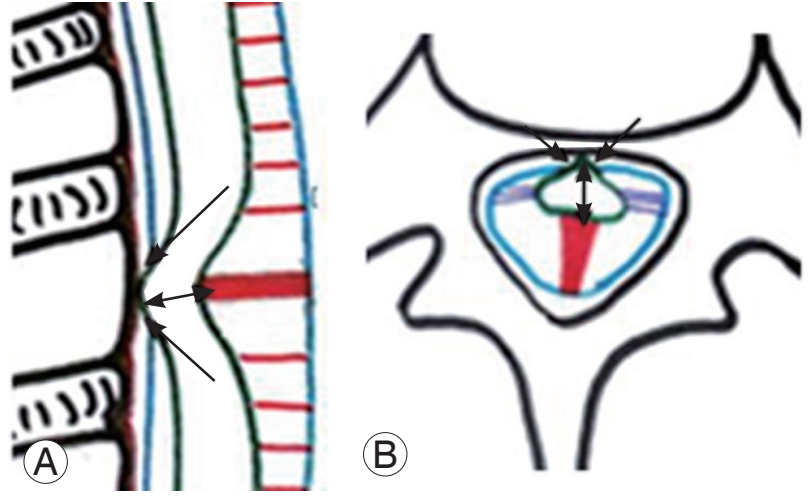

Fig. 7. (A, B) Mechanism of an anterior dural tear. The arrow indicates turbulent cerebrospinal fluid flow at the dural attachment site of the spinal cord. The double arrow indicates repeated flexion-extension movements of the spinal cord.

repetitive anterior dorsal spinal cord movement, along with other physiological events, leads to cord adhesion (Fig. 6C). The spinal cord is anteriorly displaced in an asymmetrical manner due to its adhesions to the ventral dura, which result in an absent scalpel sign for spinal cord displacement in this mechanism.

\section{Dural tear theory}

Dural inflammation at the spinal cord adhesion site produces an extrinsic pressure due to turbulent CSF flow. This, along with posterior traction exerted over the dura due to repeated extension movement of the dorsal spine, increases dural stress ultimately resulting in dural tearing (Fig. 7A, B).

Rarely, a persistent neuroenteric canal associated with anterior spina bifida, anterior meningomyeloceles, or dorsal neuroenteric cysts leads to varying severity of VDDSC; these are considered congenital causes of VDDSC $[6,10,15]$.

In our study group, patients with arachnoid webs presented without symptoms and were incidentally diagnosed. Consequently, we graded this as a milder form without significant cord compression or need for surgical intervention (grade 1). Patients with arachnoid cysts presented with milder symptoms like back pain and VDDSC, not involving the ventral dura. We considered this etiology as grade 2. In these cases, the spinal arachnoid cysts require no immediate intervention, since surgery itself carries potential complications. If symptoms worsen over time, then intervention might be required. As with cord adhesion, cord displacement also involved the ventral dura and cord herniation, in addition to dural involvement with a protruding cord. Moreover, patients with cord adhesion and herniation presented with neurological symptoms like lower limb paraparesis and bladder and bowel disturbances. We considered these entities as grades 3 and 4, respectively; these grade pathologies required immediate intervention.

Because this was a retrospective study, we were unable to correlate these pathologies with the changes in patient condition; patient follow-up was also not possible. Distribution of the VDDSC spectrum from our study group and our proposed grading system, are indicated in Table 3.

\section{Conclusions}

We proposed grouping pathologies like spinal cord herniation, cord adhesion, arachnoid cysts, and arachnoid webs that lead to VDDSC, into a single spectrum and graded them as mild, moderate, and severe forms according to the degree of displacement. 


\section{References}

1. Berg-Johnsen J, Ilstad E, Kolstad F, Zuchner M, Sundseth J. Idiopathic ventral spinal cord herniation: an increasingly recognized cause of thoracic myelopathy. J Cent Nerv Syst Dis 2014;6:85-91.

2. Lee JY, Lee JW, Lee GY, Kang HS. Focal anterior displacement of the thoracic spinal cord without evidence of spinal cord herniation or an intradural mass. Korean J Radiol 2014;15:733-8.

3. Parmar H, Park P, Brahma B, Gandhi D. Imaging of idiopathic spinal cord herniation. Radiographics 2008;28:511-8.

4. Aiyer R, El-Sherif Y, Voutsinas L. Dorsal thoracic arachnoid web presenting as neuropathic pain: 'Scalpel' sign found on MRI. Neuroradiol J 2016;29:393-5.

5. Sasani M, Ozer AF, Vural M, Sarioglu AC. Idiopathic spinal cord herniation: case report and review of the literature. J Spinal Cord Med 2009;32:86-94.

6. Rauzzino MJ, Tubbs RS, Alexander E 3rd, Grabb PA, Oakes WJ. Spinal neurenteric cysts and their relation to more common aspects of occult spinal dysraphism. Neurosurg Focus 2001;10:e2.

7. Hakky MM, Justaniah AI, David C, et al. The neuroimaging spectrum of septum posticum derangement and associated thoracic myelopathy. J Neuroimaging 2015;25:818-23.
8. Aiyer R, Voutsinas L, El-Sherif Y. An overview of arachnoid webs. J Neurol Neuromed 2016;1:66-8.

9. Taylor TR, Dineen R, White B, Jaspan T. The thoracic anterior spinal cord adhesion syndrome. Br J Radiol 2012;85:e123-9.

10. Szucs A, Horvath A, Varallyay P, et al. Spinal cord herniation: why anterior thoracic? J Neurol Neurosci 2015;6:1-5. https://doi.org/10.21767/2171-6625. S10012.

11. Najjar MW, Baeesa SS, Lingawi SS. Idiopathic spinal cord herniation: a new theory of pathogenesis. Surg Neurol 2004;62:161-70.

12. Isu T, Iizuka T, Iwasaki $Y$, Nagashima $M$, Akino $M$, Abe H. Spinal cord herniation associated with an intradural spinal arachnoid cyst diagnosed by magnetic resonance imaging. Neurosurgery 1991;29:137-9.

13. Shin JH, Krishnaney AA. Idiopathic ventral spinal cord herniation: a rare presentation of tethered cord. Neurosurg Focus 2010;29:E10.

14. Summers JC, Balasubramani YV, Chan PC, Rosenfeld JV. Idiopathic spinal cord herniation: clinical review and report of three cases. Asian J Neurosurg 2013;8:97-105.

15. Wortzman G, Tasker RR, Rewcastle NB, Richardson JC, Pearson FG. Spontaneous incarcerated herniation of the spinal cord into a vertebral body: a unique cause of paraplegia: case report. J Neurosurg 1974;41:631-5. 